\title{
Materials with graded coating under transverse loading
}

\author{
Igor A. Guz *, Maria Kashtalyan, Maryna Menshykova \\ Centre for Micro- and Nanomechanics, School of Engineering, King's College, University of Aberdeen, \\ Aberdeen AB24 3UE, Scotland, UK.
}

\begin{abstract}
In the present paper 3-D elastic deformation of a material with functionally graded coating subjected to mechanical loading is investigated. Comparative study of graded versus homogeneous coating is conducted to examine the effect of the stiffness gradient in the coating on stress and displacement fields in the material.
\end{abstract}

Copyright line will be provided by the publisher

\section{Theoretical modelling}

The concept of functionally graded materials (FGM) is currently actively explored in coating design for the purpose of increasing the resistance of coatings to functional failure. In conventional multi-layered coatings, the mismatch of thermomechanical properties between coating and substrate results in high interfacial stresses, especially in the presence of temperature gradient, which may cause cracking and debonding of the coating. Due to the importance and wide engineering applications, thermomechanical behaviour of FGM coatings has been investigated by many researchers (Guo et al [1]; Pindera et al [2]; Wang et al [3]; Liew et al [4]; Shodja et al [5]). It is worth noting that all the above investigations were carried out in the context of 2-D elasticity theory. The aim of the present paper is to investigate 3-D elastic deformation of a material with functionally graded coating subjected to mechanical loading.

A coating/substrate system considered in this paper is referred to a Cartesian co-ordinate system $O x y z$ and consists of substrate $S\left(0 \leq x \leq a, 0 \leq y \leq b, 0 \leq z \leq h^{(1)}\right)$ and the coating $C\left(0 \leq x \leq a, 0 \leq y \leq b, h^{(1)} \leq z \leq h\right)$. Both the substrate and the coating are assumed to be functionally graded, with the Poisson's ratios $\nu^{(1)}=$ const and $\nu^{(2)}=$ const, and the shear moduli that vary continuously through the thickness as

$$
G^{(k)}(z)=G^{(k)}(h) \exp \left[\gamma^{(k)}\left(\frac{z}{h}-1\right)\right], \quad k=1,2,
$$

where $\gamma^{(k)}$ are inhomogeneity parameters. The coating and the substrate are assumed to be perfectly bonded to each other, so that stress and displacement continuity conditions are satisfied at the coating/substrate interface $z=h^{(1)}$. The top and bottom surfaces of the coating/substrate system are subjected to mechanical loading. Solution to the above problem was derived by Kashtalyan and Menshykova [6].

\section{Numerical results and discussion}

In this section a comparative study of two coating/substrate systems is presented and discussed. Figure 1 shows throughthickness variation of the shear moduli. The first system (H-system) consists of homogeneous substrate with the shear modulus $G_{H}^{(1)}=$ const and homogeneous coating with the shear modulus $G_{H}^{(2)}=$ const. The second system (FGM-system) has a homogeneous substrate with the shear modulus $G_{F G M}^{(1)}=G_{H}^{(1)}$. The coating in the FGM-system is functionally graded, with the shear modulus that varies exponentially from $G_{H}^{(1)}$ to $G_{H}^{(2)}$ according to Eq.(1).
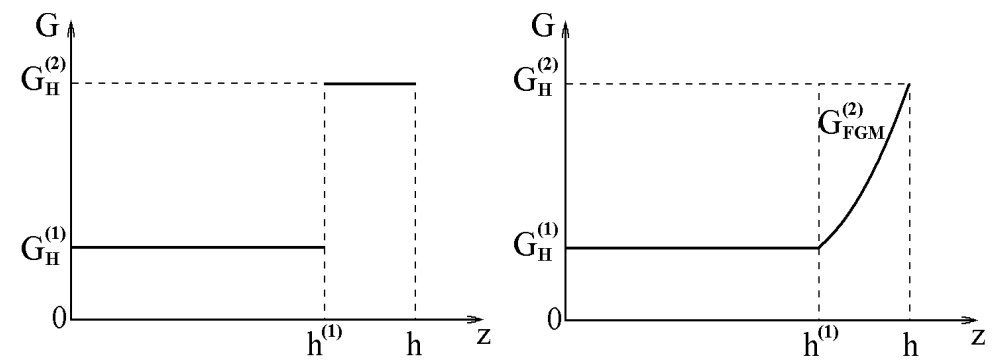

Fig. 1 Through-thickness variation of the shear moduli in two coating/substrate systems

* Corresponding author. Tel: +44 1224 272808; Fax: +44 1224 272519. E-mail address: i.guz@abdn.ac.uk 

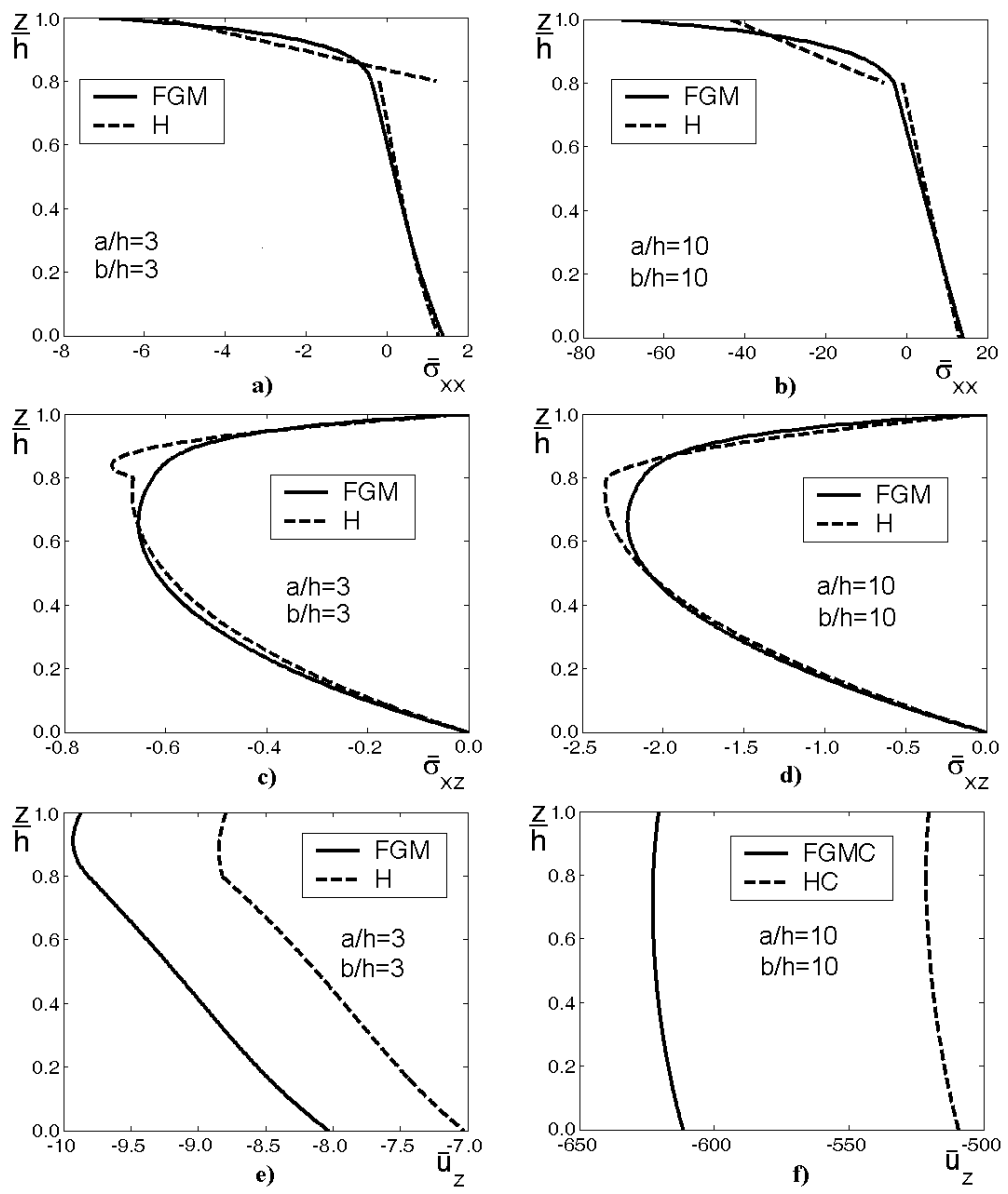

Fig. 2 Through-thickness variation of the stresses and displacements

Figure 2 shows through-thickness variations of the normalised stresses $\bar{\sigma}_{i j}=\sigma_{i j} / q$ and normalised displacements $\bar{u}_{i}=$ $G_{H}^{(2)} u_{i}(q h)^{-1}$ in two coating/substrate systems subjected to the transverse loading $q_{z z}^{(2)}(x, y)=-q \sin (\pi x / a) \sin (\pi y / b)$ on the coating surface, while bottom surface of the substrate is stress-free. The thickness of the coating is taken as $h^{(2)} / h=0.2$, and the stiffness gradient in the FGM-system $\delta^{(2)}=10$ (stiff coating protecting soft substrate). The chosen value of $\delta^{(2)}$ does not necessarily represent a certain material, but is rather used to show the effect of the coating type on the stress and displacement fields. The Poisson's ratios are taken as $\nu^{(1)}=\nu^{(2)}=0.3$. The results are shown for a thick $(a / h=b / h=3)$ and a thin $(a / h=b / h=10)$ system. Numerical results reveal that through-thickness variation of the in-plane normal stress $\bar{\sigma}_{x x}$ and the transverse shear stress $\bar{\sigma}_{x z}$ is strongly influenced by the type of the coating. In the FGM-system, as opposed to the H-system, the in-plane normal stress $\bar{\sigma}_{x x}$ is continuous across the coating/substrate interface, although its value at the top surface is higher (Fig. 2a,b). It also becomes compressive throughout the FGM coating. For the thicker H-system (Fig. 2c), the transverse shear stress $\bar{\sigma}_{x z}$ has a peak in the coating, while for FGM-systems it is close to parabolic profile, typical for homogeneous plates (Fig. 2 c,d). Through-thickness variation of the out-of-plane displacement $\bar{u}_{z}$ is highly non-linear in the thicker system (Fig. 2e), and almost constant through the thickness in the thinner system (Fig. 2f).

\section{References}

[1] Guo L.C., Wu L.Z., Ma L. Composite Structures 63(3-4), 397-406 (2004).

[2] Pindera M.J., Aboudi J., Arnold S.M. International Journal of Plasticity 21(6), 1061-1096 (2005).

[3] Wang Y.S., Huang G.Y., Gross D. Engineering Fracture Mechanics 72(12), 1942-1953 (2005).

[4] Liew K.M., Yang J., Wu Y.F. Computer Methods in Applied Mechanics and Engineering 195(9-12), 1007-1026 (2006).

[5] Shodja H.M., Haftbaradaran H., Asghari M. Composites Science and Technology 67(6), 1073-1080 (2007).

[6] Kashtalyan M., Menshykova M. International Journal of Solids and Structures 44(16), 5272-5288 (2007). 